\title{
Electron-nuclear coherent spin oscillations probed by spin dependent recombination
}

\author{
S. Azaizia, ${ }^{1}$ H. Carrère, ${ }^{1}$ J. C. Sandoval-Santana,${ }^{2}$ V. G. Ibarra-Sierra,${ }^{2}$ V. K. Kalevich,${ }^{3}$ E. \\ L. Ivchenko, ${ }^{3}$ L. A. Bakaleinikov, ${ }^{3}$ X. Marie,${ }^{1}$ T. Amand, ${ }^{1}$ A. Kunold, ${ }^{4}$ and A. Balocchi ${ }^{1, *}$ \\ ${ }^{1}$ Université de Toulouse, INSA-CNRS-UPS, LPCNO, \\ 135 avenue de Rangueil, 31077 Toulouse, France \\ ${ }^{2}$ Departamento de Física, Universidad Autónoma Metropolitana Iztapalapa, \\ Av. San Rafael Atlixco 186, Col. Vicentina, 09340 Cuidad de México, México \\ ${ }^{3}$ Ioffe Institute, 194021 St. Petersburg, Russia \\ ${ }^{4}$ Área de Física Teórica y Materia Condensada, Universidad Autónoma Metropolitana Azcapotzalco, \\ Av. San Pablo 180, Col. Reynosa-Tamaulipas, 02200 Cuidad de México, México
}

(Dated: March 1, 2022)

\begin{abstract}
We demonstrate the detection of coherent electron-nuclear spin oscillations related to the hyperfine interaction and revealed by the band-to-band photoluminescence (PL) in zero external magnetic field. On the base of a pump-probe PL experiment we measure, directly in the temporal domain, the hyperfine constant of an electron coupled to a gallium defect in GaAsN by tracing the dynamical behavior of the conduction electron spin-dependent recombination to the defect site. The hyperfine constants and the relative abundance of the nuclei isotopes involved can be determined without the need of electron spin resonance technique and in the absence of any magnetic field. Information on the nuclear and electron spin relaxation damping parameters can also be estimated from the oscillations damping and the long delay behavior.
\end{abstract}

Electron and nuclear spins of well-isolated point defects in semiconductors are excellent candidates for understanding fundamental spin-coupling mechanisms or to model quantum information processing. The coupling through hyperfine interaction (HFI) represents a key spin mechanism in semiconductor systems: responsible for creating mixed electron-nuclear spin states, it has been shown to be useful, e.g., for electron-nuclear spin transfer, in controlling electron spin coherence time of $\mathrm{P}$ donor sites in Si [1 6] and the nitrogen-vacancy centers in diamond [7-13]. The HFI is however also responsible for electron and nuclear spin relaxation and decoherence [14. Similarly to nitrogen in diamond, on one side, and to shallow defects in silicon, on the other side, interstitial $\mathrm{Ga}_{i}^{2+}$ defects in dilute nitride GaAsN [15, 16] unite the characteristics of deep and well isolated paramagnetic centers to an electrically and optically addressable semiconducting system leading, e.g., to the giant spin-dependent photocondctivity effect [17-19]. The incorporation of nitrogen in (In) GaAs to form (In) GaAsN alloys gives rise to paramagnetic interstitial centers composed of a $\mathrm{Ga}_{i}^{2+}$ atom and a single resident electron [16]. These defect sites are at the origin of a very efficient spin-dependent recombination of conduction band (CB) electrons. This has proven, for instance, to be an effective tool for generating an exceptionally high spin polarization (up to $\sim 100 \%$ ) of free and bound electrons in these nonmagnetic dilute nitrides semiconductors at room temperature 20. The nuclear spin states of these defect ensemble has been shown to be accessible via a measurement of the the circular polarization degree of band-to-band PL, while the defect nuclear spin polarization in this model system can be tuned with different excitation parameters such as pump power, the circular

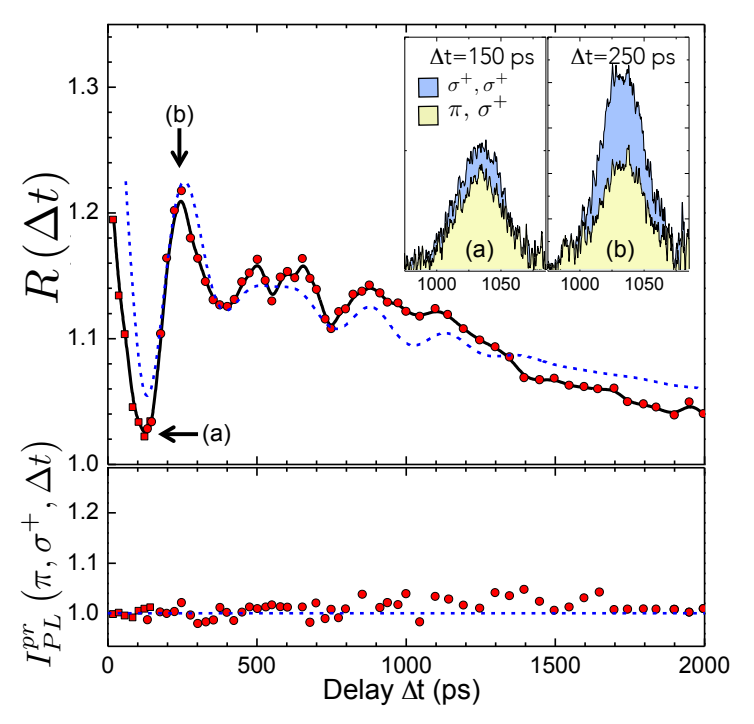

FIG. 1. (Color online) Top, symbols: The ratio of the probe pulse PL intensity under a circularly to linearly polarized pump pulse $R(\Delta t)$ measured as a function of the delay time $\Delta t$. The time origin is taken at the arrival of the pump pulse. The black line is a guide to the eye, while the blue dashed line is a fit to the data according to Eq. (7). The inset presents the PL intensities of the circularly polarized probe pulse after a circularly (blue area) or linearly (yellow area) polarized pump pulse at two different delay times (a) and (b) indicated by the arrows in the main graph. Bottom, symbols: The normalized probe pulse PL intensity after a linearly polarized pump pulse excitation. The blue dashed line presents the results of the simulation setting the polarization of the pump pulse to linear. In both figures the red circles (squares) represent the data measured with the streak camera (photodiode) set-up.

polarization degree of the incident light and through a 
weak external magnetic field 21, 23].

Optically or electrically detected magnetic resonance techniques are consistently employed for manipulating and probing the defect spins through the hyperfine interaction, or again to identify the defect chemical nature and related spin dependent recombination pathways. Here, we demonstrate the experimental implementation of a new all-optical detection scheme in zero external magnetic field based on a PL pump-probe experiment leading to the measurement, in the temporal domain, of the hyperfine constant of deep paramagnetic centers by directly tracing the hyperfine interaction dynamical features 24. The hyperfine constants, defect configuration and the relative abundance of the isotopes involved can be determined without the need of electron spin resonance techniques and in the absence of any magnetic field. Information on the nuclear and electron spin relaxation damping parameters can also be estimated from the oscillations damping and the long temporal delay behavior.

The sample under study consists of a $100 \mathrm{~nm}$ thick $\operatorname{GaAs}_{1-x} \mathrm{~N}_{x}$ epilayer $(\mathrm{x}=0.021)$ grown by molecular beam epitaxy on a (001) semi-insulating GaAs substrate and capped with $10 \mathrm{~nm}$ GaAs. The sample has been investigated at $4 \mathrm{~K}$ by the optical orientation technique which relies on the successive transfer of the angular momentum of the exciting photons, using circularly polarized light, to the photogenerated electrons [25] and finally to the $\mathrm{Ga}_{i}^{2+}$ nuclei. The excitation source is a mode-locked Ti:Sapphire laser emitting at $850 \mathrm{~nm}$ split into pump and probe pulses of equal intensity and 1.2 ps duration. Although this configuration differs from a typical pumpprobe experiments as the probe beam modifies on its turn the system similarly to the pump, this has proven to be the optimal experimental conditions to measure the HFI features. The sample is excited by focussing the pump and probe pulses to the same $50 \mu \mathrm{m}$ diameter spot. The two pulses relative delay $\Delta t$ is controlled by an optical delay line and their polarizations independently set by a system of polarization optics. In order to evidence the HFI features, we have measured the PL intensity induced by a circularly polarized probe pulse as a function of (i) the delay time between pump and probe pulses and (ii) the helicity of the pump pulse. In the following, we plot the influence of the pump pulse helicity on the probe pulse PL intensity $I_{P L}^{\mathrm{pr}}$ by computing the ratio

$$
R(\Delta t)=\frac{I_{P L}^{\mathrm{pr}}\left(\sigma^{+}, \sigma^{+}, \Delta t\right)}{I_{P L}^{\mathrm{pr}}\left(\pi, \sigma^{+}, \Delta t\right)},
$$

where the notation $\left(\sigma^{+} / \pi, \sigma^{+}, \Delta t\right)$ indicates, in arrival order, the polarization of the pump pulse, probe pulse and their respective delay. For the measurements at short delays $(\Delta t<100 \mathrm{ps}$ for which the PL intensities of the two pulses may partially overlap), the intensity of the probe pulse has been modulated by a mechanical chopper and the PL intensity of the probe pulse has

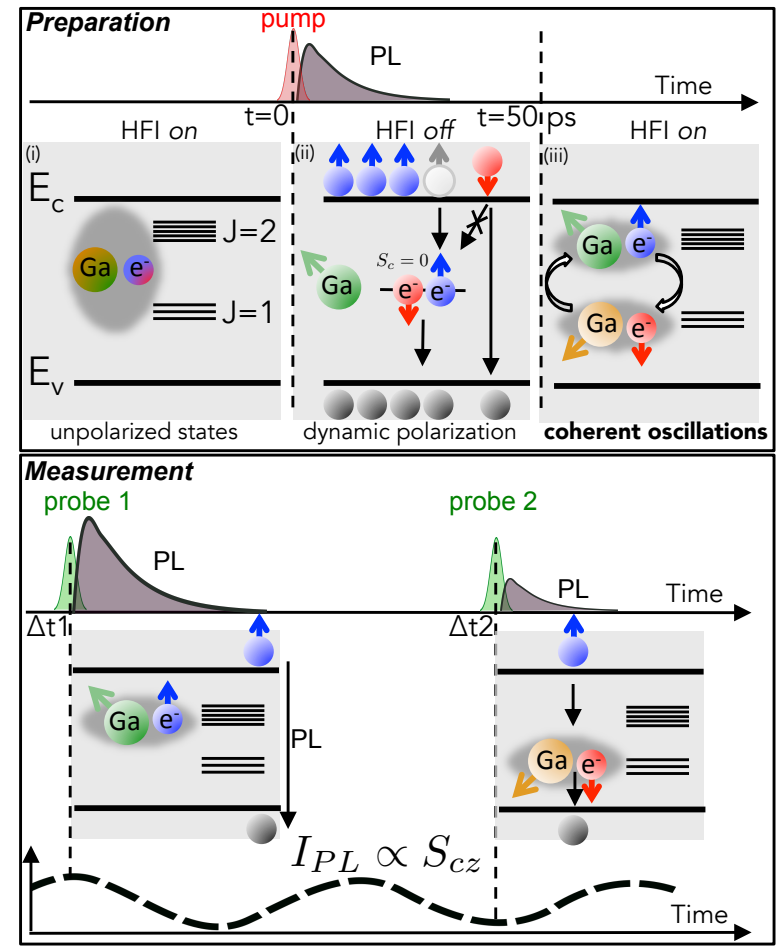

FIG. 2. (Color online) Schematic representation of the paramagnetic defect nuclear and electron spin oscillations initiated by the pump circularly polarized pulse. The defect electron arrows indicate the orientation of the average spin $S_{c z}=\left\langle\hat{S}_{c z}\right\rangle$ projections. Top: preparation of the coherent oscillations. Bottom: illustration of two extreme situations encountered by the probe beam: in the first situation (probe 1) the majority of defect electrons have the same spin orientation as the $\mathrm{CB}$ ones, preventing the capture. The PL intensity is enhanced. In the second case (probe 2), the majority of defect electrons and CB electrons have the opposite spin orientation, favoring capture: the conduction band is now depleted and the PL intensity is reduced.

been measured with a photodiode connected to a lock-in amplifier. For greater delays, a S1 photocathode streak camera coupled to an imaging spectrometer has been employed. Figure 1 (top, symbols) presents $R(\Delta t)$ measured for an excitation power $P_{e x c}=4 \mathrm{~mW}$. The trace presents an oscillating behaviour showing that $I_{P L}^{\mathrm{pr}}$ can be periodically amplified by adjusting the delay time $\Delta t$ when the pump pulse is circularly polarized. For longer delay times the oscillations lose visibility and a monotonous decrease of the amplifying effect is observed. Insets in Figure 1 display the recorded $I_{P L}^{\mathrm{pr}}$ data at two extreme points of the oscillations where the probe PL intensity is strongly increased $(\Delta t=250 \mathrm{ps})$ or only weakly modified $(\Delta t=150$ ps). Figure 1. (bottom, symbols) presents the PL intensity of the probe pulse recorded after a linearly polarized pump pulse. In this case no significant variation of the PL intensity is measured. Below we will show that the oscillating and decay features of $R(\Delta t)$ can be directly 
linked to the coherent oscillations of the electron-gallium system induced by the hyperfine-interaction.

The principle of the observation is described in terms of the model depicted in Figure 2 and based on the following considerations. The HFI hamiltonian $\hat{\mathcal{H}}_{H F I}=\mathcal{A} \hat{\boldsymbol{I}} \cdot \hat{\boldsymbol{S}}_{c}$ of gallium defects with spin $I=3 / 2$ and a singly trapped electron of spin $S_{c}=1 / 2$ (where $\mathcal{A}$ is the hyperfine interaction constant) leads to split triplet-quintet eigenstates at zero magnetic field $|J, M\rangle=\sum_{s, m} C_{s, m, M}^{\frac{1}{2}, \frac{3}{2}, J}|s, m\rangle$, where $C_{s, m, M}^{\frac{1}{2}, \frac{3}{2}, J}$ are the Clebsch-Gordan coefficients $(m= \pm 1 / 2$, $\pm 3 / 2, s= \pm 1 / 2$ are the nuclear and electron spin projections on the $z$ axis, and $M=-J, \ldots, J$ ) with total spin $J=1,2$. Before illumination by the pump pulse, the eight hyperfine states are statistically equi-populated in our experimental conditions. The preparation of the defect spin polarisation by the pump pulse proceeds as follows: A left-handed circularly polarized pumping above the band gap creates preferentially spin-up conduction band electrons (holes quickly lose their spin orientation, and are considered unpolarized [26]). The conduction band electrons are very rapidly captured by the gallium interstitial defects $\left(\tau_{e} \lesssim 10 \mathrm{ps}\right)$ forming a two-electron spin singlet [16, 27]: The hyperfine coupling is now off. It follows a fast recombination of one of the two defect electrons with an unpolarized hole. Due to the photogeneration of a $\mathrm{CB}$ electron spin polarization, the spin dependent recombination statistically drives the defect electrons to the same average spin orientation as the conduction band electrons [15, 28]. The recombination of one of the center paired electron with an unpolarized hole is fast, typically occurring on a time scale $\tau_{h} \sim 30 \mathrm{ps}[15-$ 17, and the HFI is re-enacted. At the re-establishment of the HFI the remaining electron spin state is projected onto the total spin eigenstates $|J, M\rangle$, leaving the defect system in a superposition of states between the $J=1$ and $J=2$ hyperfine levels as $\tau_{h} \cdot 2 \mathcal{A} / \hbar \ll 1$. Second, the time scale of the recombination ensures as well a relatively constant phase among the ensemble of the defect centers. At this point, the quantum system periodically oscillates between the $J=1$ and $J=2$ states which results in $S_{c z}$ oscillations between the $\pm 1 / 2$ states. Being the hyperfine interaction energy $2 \mathcal{A} \sim 15 \mu \mathrm{eV}$ [29], the defect preparation time is sizeably shorter than the oscillation period $T=h / 2 \mathcal{A} \sim 250$ ps. The probe beam (Figure 2, bottom panel) can now encounter two extreme situations. In the first case (probe 1) the majority of defect electrons have the same average spin orientation as the CB ones, preventing the capture. The PL intensity is enhanced. In the second case (probe 2), the majority of defect electrons have the opposite average spin orientation than the $\mathrm{CB}$ electrons, favoring the capture: the $\mathrm{PL}$ is now reduced as the conduction band is depleted. In principle this process would result in a partially oscillating PL intensity in a spin polarized system with sufficiently long PL characteristic decay time $\tau_{P L}$ in a single pulse experiment. Here, being the PL decay time shorter than the oscillation period T, a pump-probe PL technique, employing a second pulse, samples in time the coherent oscillation of the hyperfine system. It is important to note that longer PL decay times $\tau_{P L}$ (as can be obtained by higher power excitation in this systems [30]) are here not desirable as this will smear the initialization of the oscillation leading to faster dephasing. If however the pump pulse is linearly polarized, no dynamical polarization can occur and the probe pulse spin dependent recombination will be insensitive to the delay time. A key feature is that this all-optical approach does not require any external magnetic field which might modify the spin relaxation damping parameters. Let us now turn our attention to the kinetics of photoelectrons excited by the probe pulse in the conduction band. We can get a qualitative and analytical understanding of the oscillating behavior of $R(\Delta t)$ according to the following argument. Neglecting the electron spin relaxation, the $\mathrm{CB}$ electron rate equations can be described by

$$
\begin{aligned}
& \frac{d n_{+}}{d t}+2 c_{n} n_{+} N_{-}+\gamma_{r} n_{+} p=0, \\
& \frac{d n_{-}}{d t}+2 c_{n} n_{-} N_{+}+\gamma_{r} n_{-} p=0,
\end{aligned}
$$

where $n_{ \pm}(t)$ are the densities of the conduction photoelectrons with spin up $(+)$ and down $(-)$ excited by the probe pulse arriving with the time delay $\Delta t, c_{n}$ is the constant of the conduction-electron trapping rate by paramagnetic centers, $p$ is the hole concentration (due to rapid spin relaxation the holes are unpolarized), $\gamma_{r}$ is the bimolecular recombination constant, and $N_{ \pm}$are the concentrations of single-electron defects with the electron spin $\pm 1 / 2$ with $N_{+}+N_{-}=N_{1}$. Due to the prior pump pulse $N_{+}$and $N_{-}$are different if the pump is circularly polarized and coincide for the linearly polarized pump excitation. Since (i) the electron capture is much more effective as compared to the interband recombination and (ii) $N_{ \pm}$vary slowly within the capture times $\left(c_{n} N_{ \pm}\right)^{-1}$, the time dependence of $n_{ \pm}$is described by $n_{ \pm}^{\mathrm{pr}} \exp \left[-2 c_{n} N_{\mp}(\Delta t) t\right]$, where $n_{ \pm}^{\mathrm{pr}}$ are the electron densities injected by the probe pulse. For a sufficiently weak photoexcitation the measured ratio (1) is described by

$$
R(\Delta t)-1 \propto\left(n_{+}^{\mathrm{pr}}-n_{-}^{\mathrm{pr}}\right)\left[N_{+}(\Delta t)-N_{-}(\Delta t)\right] .
$$

For circularly polarized pump pulses the values $N_{ \pm}(\Delta t)$ consist of the oscillating and non-oscillating parts

$$
N_{ \pm}(\Delta t)=N_{ \pm, 0} \pm \frac{\delta N}{2} \cos (\Omega \Delta t),
$$

where $\hbar \Omega=2 \mathcal{A}$ is the hyperfine splitting between the electron-nuclear spin quintet and triplet with the angular momenta $J=2$ and $J=1$, respectively.

The oscillating time behaviour of $N_{ \pm}(\Delta t)$ can be understood in terms of the spin-density-matrix approach. 
In equilibrium the spin density of single-electron defects, $\rho_{J^{\prime}, M^{\prime} ; J, M}$, is diagonal with equally populated sublevels: $\rho_{J^{\prime}, M^{\prime} ; J, M}=\left(N_{1} / 8\right) \delta_{J^{\prime} J} \delta_{M^{\prime} M}$. The pump pulse generates $\mathrm{CB}$ photoelectrons with densities $n_{ \pm}^{\mathrm{pm}}$ which are immediately captured by single-electron defects according to Eqs. (2) and form the electron pair states with density $N_{2}=n_{+}^{\mathrm{pm}}+n_{-}^{\mathrm{pm}}$. The remaining single-electron defects acquire spin polarization. Immediately after the pulse, i.e. at $\Delta t=0$, one has $N_{ \pm}(0)=N_{c} / 2-n_{\mp}^{\mathrm{pm}}$, where $N_{c}=N_{1}+N_{2}$ is the total density of the deep paramagnetic centers. This equation can be rewritten as

$$
N_{ \pm}(0)=\sum_{m} \rho_{ \pm \frac{1}{2}, m ; \pm \frac{1}{2}, m}(0)
$$

in terms the spin-density matrix $\rho_{s^{\prime}, m^{\prime} ; s, m}(0)=$ $\delta_{s^{\prime} s} \delta_{m^{\prime} m}\left(N_{c} / 8-n_{-s}^{\mathrm{pm}}\right)$ taken in the basis $|s, m\rangle$. In the basis $|J, M\rangle$ this equation is rewritten as

$$
N_{ \pm}(0)=\sum_{m J^{\prime} J} D_{J^{\prime}, J ; \pm 1 / 2, m} \rho_{J^{\prime}, m \pm \frac{1}{2} ; J, m \pm \frac{1}{2}}(0)
$$

where

$$
D_{J^{\prime}, J ; s, m}=C_{s, m, s+m}^{\frac{1}{2} \frac{3}{2} J^{\prime}} C_{s, m, s+m}^{\frac{1}{2} \frac{3}{2} J}
$$

and the Clebsch-Gordan coefficients $C_{s m M}^{\frac{1}{2} \frac{3}{2} J}$ relate the standard bases $|s, m\rangle$ and $|J, M\rangle$. The components $\rho_{J^{\prime}, M ; J ; M}(0)$ can be readily expressed via $N_{c}, n_{+}^{\mathrm{pm}}$ and $n_{-}^{\mathrm{pm}}$, among them there are those with $J^{\prime}=J$ and $J^{\prime} \neq J$. Neglecting the spin relaxation we have

$$
\rho_{J^{\prime}, M ; J, M}(\Delta t)=\rho_{J^{\prime}, M ; J, M}(0) \mathrm{e}^{-\mathrm{i} \Omega\left(J^{\prime}-J\right) \Delta t} .
$$

Therefore, the oscillating part of Eq. (4) is contributed from the off-diagonal spin-matrix components with $J^{\prime} \neq$ $J$. A straightforward calculation gives

$$
N_{+, 0}-N_{-, 0}=\frac{3}{8}\left(n_{+}^{\mathrm{pm}}-n_{-}^{\mathrm{pm}}\right), \delta N=\frac{5}{8}\left(n_{+}^{\mathrm{pm}}-n_{-}^{\mathrm{pm}}\right) .
$$

The last terms in the left-hand sides of Eqs. (2) describe the radiative recombination and the PL intensity. Retaining all the factors we can present Eq. (3) in the final form

$$
R(\Delta t)-1=\alpha+\beta \cos (\Omega \Delta t)
$$

where the coefficients $\alpha$ and $\beta$ are respectively given by $P_{i}\left(N_{+, 0}-N_{-, 0}\right) / N_{c}$ and $P_{i} \delta N / N_{c}$ with $P_{i}$ being the initial degree of the pump-induced spin polarization $\left(n_{+}^{\mathrm{pm}}-n_{-}^{\mathrm{pm}}\right) /\left(n_{+}^{\mathrm{pm}}+n_{-}^{\mathrm{pm}}\right)$. In the presence of two isotopes, as is the case for gallium atoms, the cosine function in Eq. (6) should be replaced by a sum of two cosine functions with the frequencies $\Omega_{1}, \Omega_{2}$ and relative abundances $f_{1}, f_{2}$. Allowance for the spin relaxation results in a multiplication of $\alpha$ by $\exp \left(-\Delta t / \tau_{s c}\right)$ and of $\beta$ by

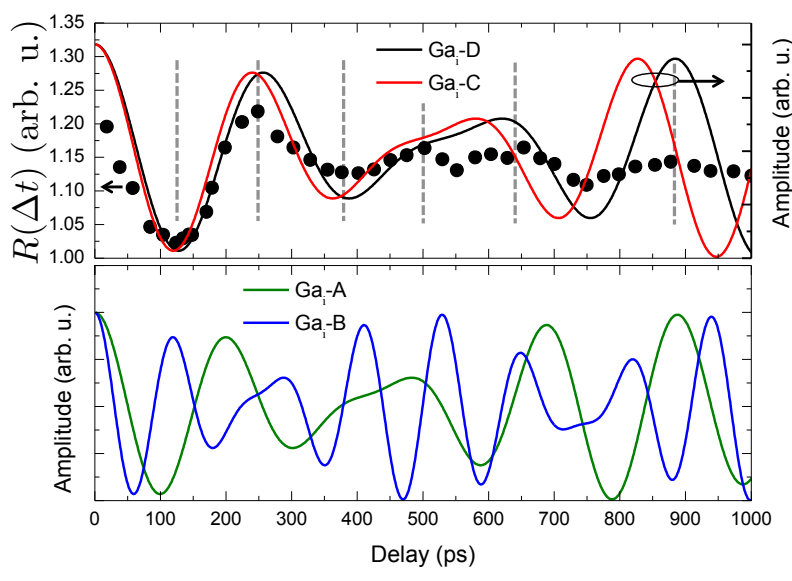

FIG. 3. (Color online) Detail of the experimental data for short delays (circles) superimposed to simple cosine beat patterns (solid lines) at the frequencies of the different interstitial configurations. The vertical dashed lines indicate the position of the extrema of the experimental data.

$\exp \left(-\Delta t / T_{2}\right)$, where $\tau_{s c}$ is the bound-electron spin relaxation time and $T_{2}$ is the decoherence time of electronnuclear spin oscillation which is affected by both homogeneous relaxation processes and inhomogeneous broadening of the hyperfine splitting. Thus the experimentally determined $R(\Delta t)$ can be quantitatively compared with

$$
\begin{aligned}
R(\Delta t) & -1=\alpha \mathrm{e}^{-\Delta t / \tau_{s c}} \\
& +\beta \mathrm{e}^{-\Delta t / T_{2}}\left[f_{1} \cos \left(\Omega_{1} \Delta t\right)+f_{2} \cos \left(\Omega_{2} \Delta t\right)\right],
\end{aligned}
$$

where the positive values $f_{1}$ and $f_{2}$ are normalized by the condition $f_{1}+f_{2}=1$. According to the mechanism described, the circularly polarised probe pulse will sample the oscillating behaviour of the hyperfine coupling instantaneously and at different delays. We obtain a modulation of the probe beam PL intensity directly tracing the hyperfine interaction in the time domain. In the case of gallium atoms, the two stable isotopes ${ }^{69} \mathrm{Ga}$ and ${ }^{71} \mathrm{Ga}$ have the relative abundances $f_{1}=0.6018$ and $f_{2}=0.3982$ respectively, and their hyperfine constants differs by a factor $\mathcal{A}_{2} / \mathcal{A}_{1}=1.27$. The hyperfine constant (Table I 29]) will however depend on the particular defect location which is determined mainly by growth and annealing conditions. Figure 3 details $R(\Delta t)$ for short delays superposed to a simple beating pattern composed of a sum of simple cosines functions at the frequencies of the different gallium interstitial locations as reported in Table 1. The experimental data present a first destructive interference pattern in the 500 ps to 600 ps range. This rules out the occurrence of $\mathrm{Ga}_{i}-\mathrm{A}$ and $\mathrm{Ga}_{i}$-B as their higher oscillating frequencies are incompatible with the experimental observation. $\mathrm{Ga}_{i}-\mathrm{C}$ and $\mathrm{Ga}_{i}$-D present very similar hyperfine constant which causes the beating pattern to dephase slightly. Nevertheless, the experimental data presented in Figure 3 (top) allow us to unambiguously identify $\mathrm{Ga}_{i}$-D as the dominant interstitial config- 


\begin{tabular}{lccccc}
$\mathrm{Ga}_{i}$ location & A & B & C & D \\
\hline & & & & \\
\hline $\mathcal{A}_{1}\left({ }^{69} \mathrm{Ga}\right)\left(\times 10^{-4}\right.$ & $\left.\mathrm{cm}^{-1}\right)$ & 745 & 1230 & 620 & 580 \\
$\mathcal{A}_{2}\left({ }^{71} \mathrm{Ga}\right)\left(\times 10^{-4} \mathrm{~cm}^{-1}\right)$ & 968.5 & 1562 & 787.4 & 736.6
\end{tabular}

TABLE I. The hyperfine interaction constants for the two naturally stable isotopes of gallium in the four different interstitial configurations occurring in dilute nitrides (In)GaAsN [29].

uration.

The dashed line in Figure 1 (top) represents a fit to the data using Eq. (7), the hyperfine constants of gallium D interstitial defects and setting the pump pulse polarization to circular. The best results are obtained with $\tau_{s c}=1700 \mathrm{ps}, T_{2}=350 \mathrm{ps}$. This proves that a precise determination of the defect nature and configuration can be obtained by this PL pump and probe scheme. The mechanisms responsible for the coherence loss can be multiple. First of all, the measurement maps the coherent oscillations of the ensemble of Ga centers present under the excitation spot whose intensity strongly varies from the excitation spot center to the edge. Second, the HFI sampling cannot be considered as strictly instantaneous but averaged over the $\mathrm{CB}$ electron lifetime $\tau_{P L}$, allowing us only to infer a minimum limit for the coherence time decay. Third, the stochastic nature of the the trapping and recombination can also be ascribed as a possible source of coherence loss. Finally, there might be some inhomogeneities from center to center resulting in slight fluctuations of the hyperfine coupling constant. The dotted line in Figure 1 (bottom) reports the results of the simulation setting the first pulse polarization to linear. As expected from the previous discussion, $N_{+}$and $N_{-}$are now identical and no oscillations are observed.

In conclusion, we have demonstrated a possibility of measuring the electron-nuclear spin oscillations related to the hyperfine interaction in dilute nitride semiconductor paramagnetic centers by monitoring the band to band PL in the absence of any magnetic field. The hyperfine constants and the relative abundances of the nuclei involved can be unambiguously determined without the need of electron spin resonance techniques. Information on the nuclear and electron spin relaxation damping parameters of the paramagnetic center can also be estimated from the oscillations damping and the long time delay behavior. This zero magnetic field detection scheme based solely on the spin dependent recombination should be applicable to other materials besides GaAsN, like 2D crystals as, for instance, paramagnetic centers in h-BN 31.

We acknowledge funding from LIA CNRS-Ioffe RAS ILNACS. E.L.I. thanks the RFBR (Grant No. 17-0200383). V.K.K. acknowledges the financial support of the Government of Russia (Project No. 14.Z50.31.0021).
A.K. gratefully appreciates the financial support of Departamento de Ciencias Bàsicas UAM-A grant numbers 2232214 and 2232215 . J.C.S.S. and V.G.I.S. would like to acknowledge the support received from the Becas de Posgrado UAM scholarship numbers 2151800745 and 2112800069. X.M. also thanks Institut Universitaire de France. This work was supported by Programme Investissements d'Avenir under the program ANR-11IDEX-0002-02, reference ANR-10-LABX-0037-NEXT.

* andrea.balocchi@insa-toulouse.fr

[1] B. E. Kane, Nature 393, 133 (1998)

[2] J. J. Pla, K. Y. Tan, J. P. Dehollain, W. H. Lim, J. J. L. Morton, D. N. Jamieson, A. S. Dzurak, and A. Morello, Nature 489, 541 (2012)

[3] T. D. Ladd, D. Maryenko, Y. Yamamoto, E. Abe, and K. M. Itoh, Phys. Rev. B 71, 014401 (2005).

[4] A. Laucht, R. Kalra, J. T. Muhonen, J. P. Dehollain, F. A. Mohiyaddin, F. Hudson, J. C. McCallum, D. N. Jamieson, A. S. Dzurak, and A. Morello, Appl. Phys. Lett. 104, 092115 (2014).

[5] W. Yao, R.-B. Liu, and L. J. Sham, Phys. Rev. B 74, 195301 (2006)

[6] V. Ivády, K. Szász, A. L. Falk, P. V. Klimov, D. J. Christle, E. Janzén, I. A. Abrikosov, D. D. Awschalom, and A. Gali, Phys. Rev. B 92, 115206 (2015)

[7] D. D. B. Rao, S. Yang, and J. Wrachtrup, Phys. Rev. B 92, 081301 (2015).

[8] S. Zaiser, T. Rendler, I. Jakobi, T. Wolf, S.-Y. Lee, S. Wagner, V. Bergholm, T. Schulte-Herbrggen, P. Neumann, and J. Wrachtrup, Nat. Comm. 7, 12279 (2016)

[9] A. Auer and G. Burkard, Phys. Rev. B 93, 035402 (2016).

[10] T. van der Sar, Z. H. Wang, M. S. Blok, H. Bernien, T. H. Taminiau, D. M. Toyli, D. A. Lidar, D. D. Awschalom, R. Hanson, and V. V. Dobrovitski, Nature 484, 82 (2012)

[11] N. Mizuochi, P. Neumann, F. Rempp, J. Beck, V. Jacques, P. Siyushev, K. Nakamura, D. J. Twitchen, H. Watanabe, S. Yamasaki, F. Jelezko, and J. Wrachtrup, Phys. Rev. B 80, 041201 (2009)

[12] J. R. Maze, J. M. Taylor, and M. D. Lukin, Phys. Rev. B 78, 094303 (2008)

[13] J. J. Pla, F. A. Mohiyaddin, K. Y. Tan, J. P. Dehollain, R. Rahman, G. Klimeck, D. N. Jamieson, A. S. Dzurak, and A. Morello, Phys. Rev. Lett. 113, 246801 (2014)

[14] B. Urbaszek, X. Marie, T. Amand, O. Krebs, P. Voisin, P. Maletinsky, A. Högele, and A. Imamoglu, Rev. Mod. Phys. 85, 79 (2013).

[15] V. K. Kalevich, E. L. Ivchenko, M. M. Afanasiev, A. Y. Shiryaev, A. Y. Egorov, V. M. Ustinov, B. Pal, and Y. Masumoto, JETP Lett. 82, 455 (2005)

[16] X. J. Wang, I. A. Buyanova, F. Zhao, D. Lagarde, A. Balocchi, X. Marie, C. W. Tu, J. C. Harmand, and W. M. Chen, Nature Mat. 8, 198 (2009).

[17] D. Lagarde, L. Lombez, X. Marie, A. Balocchi, T. Amand, V. K. Kalevich, A. Shiryaev, E. Ivchenko, and A. Egorov, Phys. Stat. Sol. (a) 204, 208 (2007).

[18] F. Zhao, A. Balocchi, A. Kunold, J. Carrey, H. Carrre, T. Amand, N. Ben Abdallah, J. C. Harmand, and 
X. Marie, Appl. Phys. Lett. 95, 241104 (2009).

[19] A. Kunold, A. Balocchi, F. Zhao, T. Amand, N. B. Abdallah, J. C. Harmand, and X. Marie, Phys. Rev. B 83, $165202(2011)$

[20] V. K. Kalevich, A. Y. Shiryaev, E. L. Ivchenko, A. Y. Egorov, L. Lombez, D. Lagarde, X. Marie, and T. Amand, JETP Lett. 85, 174 (2007).

[21] V. K. Kalevich, M. M. Afanasiev, A. Y. Shiryaev, and A. Y. Egorov, Phys. Rev. B 85, 035205 (2012).

[22] V. K. Kalevich, M. M. Afanasiev, A. Y. Shiryaev, and A. Y. Egorov, JETP Lett. 96, 567 (2013)

[23] E. L. Ivchenko, L. A. Bakaleinikov, M. M. Afanasiev, and V. K. Kalevich, Phys. Sol. St. 58, 1539 (2016).

[24] V. G. Ibarra-Sierra, J. C. Sandoval-Santana, S. Azaizia, H. Carrre, L. Bakaleinikov, V. K. Kalevich, E. L. Ivchenko, X. Marie, T. Amand, A. Balocchi, and A. Kunold, arXiv:1611.04200 (2016).

[25] F. Meier and B. P. Zakharchenya, Optical Orientation (Elsevier Science Ltd, 1984).
[26] D. J. Hilton and C. L. Tang, Phys. Rev. Lett. 89, 146601 (2002)

[27] V. K. Kalevich, A. Y. Shiryaev, E. L. Ivchenko, M. M. Afanasiev, A. Y. Egorov, V. M. Ustinov, and Y. Masumoto, Phys. B 404, 4929 (2009).

[28] C. Sandoval-Santana, A. Balocchi, T. Amand, J. C. Harmand, A. Kunold, and X. Marie, Phys. Rev. B 90, $115205(2014)$

[29] X. J. Wang, Y. Puttisong, C. W. Tu, A. J. Ptak, V. K. Kalevich, A. Y. Egorov, L. Geelhaar, H. Riechert, W. M. Chen, and I. A. Buyanova, Appl. Phys. Lett. 95, 24190 (2009).

[30] F. Zhao, A. Balocchi, G. Truong, T. Amand, X. Marie, X. J. Wang, I. A. Buyanova, W. M. Chen, and J. C. Harmand, J. of Phys.: Cond. Mat. 21, 174211 (2009).

[31] T. T. Tran, K. Bray, M. J. Ford, M. Toth, and I. Aharonovich, Nat. Nanotech. 11, 37 (2016). 